\title{
DIRECTIONAL CURRENTS IN NOCTURNAL E-REGION LAYERS
}

\author{
H. RISHBETH* and J. C. G. WALKEK $\dagger$ \\ *Rutherford Appleton Laboratory, Chilton, Didcot, Oxon OX11 0QX, U.K. \\ †Space Physics Research Laboratory, University of Michigan, Ann Arbor, MI 48109, U.S.A.
}

\section{(Received 25 September 1981)}

\begin{abstract}
In the mid-latitude $E$-region, the wind-shear mechanism produces thin ionized layers at levels where the vertical ion velocity is zero. We show that such layers conduct electric current only towards the magnetic equator, and not in the zonal direction. We surmise that this property may influence the electric field distribution in the nocturnal ionosphere, and possibly also the coupling between ion drifts and neutral air winds in the $F$-region. Detailed case studies of nocturnal layers located near the peak of ion Pedersen conductivity (around $130 \mathrm{~km}$ ) are needed to test this idea.
\end{abstract}

\section{INTRODUCTION}

The wind-shear theory of sporadic $E$ has been quite successful in describing the formation and behaviour of certain thin layers in the ionospheric $E$-region. The theory predicts that, under the influence of the geomagnetic field, plasma will be concentrated at or near levels where the zonal wind pattern produces "convergent nulls" in the vertical ion velocity. Within the complex tidal structure of thermospheric winds, the nulls move progressively downwards, imposing their vertical motion on the layers of plasma by the so-called "corkscrew effect". The wind-shear theory, and related topics such as the metallic ion composition of Es-layers and the corkscrew mechanism, have been extensively discussed in the literature (Whitehead, 1961; Axford, 1961; Axford and Cunnold, 1966; Whitehead, 1970, etc.). Experimental data on the relationship between winds and plasma layers nave been obtained by rocket-borne techniques (e.g. Fujitaka et al., 1971; Rees et al., 1976) and ground-based radars. The most detailed and systematic case studies have been made with the Arecibo incoherent scatter radar (e.g. Rowe, 1974; Harper et al., 1975; Shen et al., 1976; Miller and Smith, 1978; Trost, 1979; Mathews and Bekeny, 1979). Many of the observed plasma layers are quite long-lived and stable, and should be termed "intermediate layers" or "night $E$ " rather than sporadic $E$. Our discussion applies to any noctur- nal layer, sporadic or otherwise, produced by the wind-shear mechanism. The observations of Harper et al. (1975) and the theoretical calculations of Macleod et al. (1975) and Mathews and Bekeny (1979) all illustrate the way in which the layers follow the "convergent nulls" of vertical ion velocity.

In Section 2 we develop the appropriate equations for ion and electron motions and electric currents. We then show in Section 3 that levels of zero vertical ion velocity are also levels of zero zonal electric current. Electric currents at these levels are therefore meridional, in fact equatorward. If the concentrations at these levels represent an appreciable fraction of the total $E$ region plasma content, as may be the case at night (Section 4) the total $E$-region current will have a meridional bias. This bias might be reflected in the degree to which $F$-region polarization fields are short-circuited in the night $E$-region, and hence in the coupling of neutral air and plasma motions in the $F$-region (Section 5).

\section{EQUATIONS FOR THE CURRENT FLOWING IN A THIN LAYER}

To demonstrate the directional nature of the current in a thin layer at a level where the vertical ion velocity is zero, we start with the familiar equations of motion for ions and electrons in the ionosphere, in the presence of an electrostatic 
polarization field $\mathbf{E}$, normal to the geomagnetic flux density $\mathbf{B}$, and a neutral air wind $\mathbf{U}$. We neglect gravity and pressure gradients which, though they influence the detailed structure of the layers, are not important to the present discussion. We assume for simplicity that there is a single ion species with concentration $N$, mass $m_{\mathrm{i}}$, charge $+e$, collision frequency $\nu_{i}$ with neutral particles and gyrofrequency $\omega_{\mathrm{i}}=B e / m_{\mathrm{i}}$. The assumption of a single ion species ought to be dispensed with in future work, since a mixture of metallic and gaseous ions may exist in the layers under discussion. The height $h_{\mathrm{i}}$ at which $\nu_{\mathrm{i}}=\omega_{\mathrm{i}}$ is around $130 \mathrm{~km}$.

Following Rishbeth and Garriott (1969) (pp. 132135) we write the force equation for ions in terms of a tensor mobility [k] of which the components are the "direct", "Pedersen" and "Hall" ion mobilities (velocity/force ratios) $k_{0}, k_{1}, k_{2}$. If $\mu=$ $\omega_{\mathrm{i}} / \nu_{\mathrm{i}}=B e / m_{\mathrm{i}} \nu_{\mathrm{i}}$ then

$$
\begin{gathered}
k_{0}=\frac{1}{m_{\mathrm{i}} \nu_{\mathrm{i}}}, \quad k_{1}=\frac{1}{B e} \frac{\mu}{1+\mu^{2}}, \\
k_{2}=\frac{1}{B e} \frac{\mu^{2}}{1+\mu^{2}} .
\end{gathered}
$$

We use two different right-handed sets of directions, the geometric set $x$, magnetic east; $y$, magnetic north; $z$ (or $h$ ), vertically up and the field perpendicular/parallel set $x$, magnetic east; $n$, equatorward and downward at angle $\left(\frac{1}{2} \pi-I\right)$; $\|$, field parallel, i.e. northward and downward at angle $I$; the dip angle $I$ being positive (negative) in the northern (southern) hemisphere. The latter set is useful for the electrostatic field $\mathbf{E}$ which is essentially field-perpendicular, the former set for wind $\mathbf{U}$ and current $\mathbf{j}$ which are essentially horizontal. The ion drift velocity given by

$$
\mathbf{V}_{\mathrm{i}}=[\mathbf{k}] \cdot\left(e \mathbf{E}+m_{\mathrm{i}} \nu_{\mathrm{i}} \mathbf{U}\right)
$$

has components

$$
\begin{aligned}
& V_{\mathrm{i} x}=k_{1}\left(e E_{x}+m_{\mathrm{i}} \nu_{\mathrm{i}} U_{x}\right)+k_{2}\left(e E_{n}+m_{\mathrm{i}} \nu_{\mathrm{i}} U_{n}\right) \\
& V_{\mathrm{i} n}=-k_{2}\left(e E_{x}+m_{i} \nu_{i} U_{x}\right)+k_{1}\left(e E_{n}+m_{\mathrm{i}} \nu_{\mathrm{i}} U_{n}\right) \\
& V_{\mathrm{i} \|}=k_{0}\left(e E_{\|}+m_{\mathrm{i}} \nu_{\mathrm{i}} U_{\|}\right) .
\end{aligned}
$$

For the electrons the gyrofrequency so greatly exceeds the collision frequency at all $E$ - and $F$ region $r$ ights that only the "Hall" equations need to be c rsidered.

$$
V_{e x}=E_{n} / B ; \quad V_{e n}=-E_{x} / B .
$$

Owing to their excellent field-aligned mobility, electrons can move freely along the magnetic field to neutralize any field-aligned component $\mathbf{F}_{\|}$. Furthermore, the bounded nature of the ionosphere inhibits any substantial vertical current $j_{z}$, the clectric ficld being so adjusted that $j_{2}=0$. This assertion holds well enough even allowing for field-aligned currents caused by asymmetries in the global dynamo system [e.g. Rishbeth and Garriott (1969), p. 238]. There may be additional localized field-aligned currents at the periphery of the layers discussed in this paper; but within the layers we may take

$$
j_{x} \equiv-j_{\|} \sin I-j_{n} \cos I=0
$$

and the northward horizontal current is then given by

$$
j_{y} \equiv j_{\|} \cos I-j_{n} \sin I=-j_{n} / \sin I .
$$

It follows that the electric current density

$$
\mathbf{j}=\operatorname{Ne}\left(\mathbf{V}_{\mathrm{i}}-\mathbf{V}_{\mathrm{e}}\right),
$$

has components

$$
\begin{aligned}
j_{x}= & \frac{N e / B}{1+\mu^{2}}\left[\mu E_{x}-E_{n}+B U_{x}-\mu B U_{y} \sin I\right] \\
-j_{y}= & \frac{N e / B}{1+\mu^{2}}\left[E_{x}+\mu E_{n}-\mu B U_{x}\right. \\
& \left.\quad-B U_{y} \sin I\right] \operatorname{cosec} I .
\end{aligned}
$$

\section{THE DIRECTIONAL EFFECT}

To proceed further we make use of the finding (which is both observational and theoretical) that the thin layers occur at or near levels where the vertical ion velocity is zero. This condition may be written as

$$
V_{\mathrm{i} z} \equiv-V_{\mathrm{i} \|} \sin I-V_{\mathrm{in}} \cos I=0 .
$$

From (1, 4 and 5) we can obtain an expression for $V_{\mathrm{iz}}$ in terms of $\mu-\omega_{\mathrm{i}} / \nu_{\mathrm{i}}$ and components of $\mathrm{U}$ and E. Since $\mathbf{E}$ is essentially field-perpendicular we retain its components in the $(x, n, \|)$ set of directions; but we convert the wind components to the $(x, y, z)$ set. We find that

$$
\begin{gathered}
V_{\mathrm{i} z}=\frac{\mu / B}{1+\mu^{2}}\left[\mu E_{x}-E_{n}+B U_{x}-\mu B U_{y} \sin I\right] \cos I \\
-\frac{e E_{\|}}{m_{\mathrm{i}} \nu_{\mathrm{i}}} \sin I+\frac{1+\mu^{2} \sin ^{2} I}{1+\mu^{2}} U_{z}
\end{gathered}
$$


The square bracket in equation (13) is the same as in (10). If now we assume $E_{\|}=0$ (as previously mentioned) and furthermore assume that the vertical wind $U_{z}$ is small compared with the horizontal components $U_{x}, U_{y}$ (as seems reasonable) we neglect the last two terms in equation (13) so that

$$
j_{\mathrm{x}}=N e V_{\mathrm{i} z} / \mu \cos I .
$$

This shows that if a layer forms at the level where $V_{i z}=0$, as observed, it will carry no east-west or $x$-component of current. The current in the layer is therefore purely meridional. The conditions under which this happens depends on the relative magnitudes of the terms in the square bracket of equation (10) or (13). The electric field components $E_{x}, E_{h}$ may be assumed height-independent, but the wind components $U_{x}, U_{y}$ are part of a tidal system in which the wind vector rotates with height. Provided the electric field is not too large in relation to the wind, there will be levels at which the square bracket vanishes and hence
$V_{\mathrm{i} z}=0$. For the moment, take $E_{x}=E_{n}=0$ in which case $V_{\mathrm{i} z}$ is simply given by

$$
V_{\mathrm{i} z}=\frac{\mu \cos I}{1+\mu^{2}}\left[U_{x}-\mu U_{y} \sin I\right]
$$

with $j_{x}$ proportional to $V_{\mathrm{i} z}$, as in (14), while the northward current from (11) is now

$$
j_{y}=\frac{N e}{1+\mu^{2}}\left[\mu U_{x} \operatorname{cosec} I+U_{y}\right]
$$

At Arecibo Harper (1977) found that the wind vector in the $E$ - and lower $F$-regions rotates clockwise with increasing height. Though the $E$ region wind measurements were confined to daytime, the dominant semidiurnal wind system is believed responsible for producing the night-time layers. In such a rotating wind system the sign of $V_{i z}$ oscillates with height in the manner shown in Figure 1, for the three regimes $\mu \gg 1, \mu \sim 1$ and $\mu \ll 1$. In reality these regimes are not distinct but

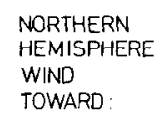

\begin{tabular}{llll} 
PATTERN OF VERTICAL ION & DRIFT & SOUTHERN \\
\hline LOWER & TRANSITION & MIDDLE & HEMISPHERE \\
F REGION & $h-130 \mathrm{~km}$ & E REGION & WIND \\
$\left.\left.\omega_{i}\right\rangle\right\rangle v_{i}$ & $\omega_{i}-v_{i}$ & $\omega_{i}\left\langle\left\langle v_{i}\right.\right.$ & TOWARD :
\end{tabular}

NE

$\mathrm{N}$

NW

W

SW

$S$

SE

E

NE

N

NW

w

$-\theta$

$-$
D

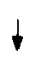

c

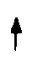

D

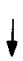

$\pi / 2$ $\omega_{i}-v_{i}$

D

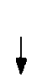

c

$c$

$\uparrow$

D
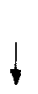

$\pi / 4$

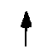

D S

SW

$\checkmark \quad W$

NW

C N

NE

1 E

D S

SW

b $\quad$ W

$0 \quad \bar{\theta}$
SE
W

NW

\section{NE}

SE

5

W

$\bar{\theta}$

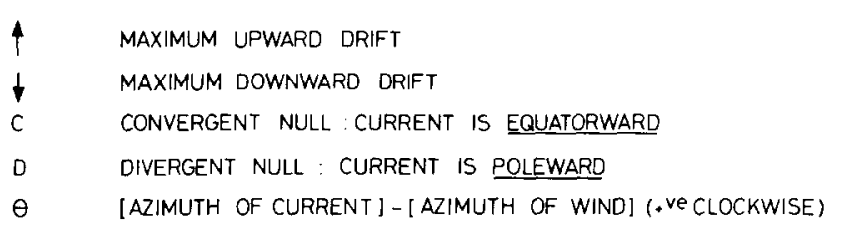

EACH COLUMN REPRESENTS $1 \frac{1}{2}$ VERTICAL WAVELENGTHS OF THE WIND PATTERN

FIG. 1. WIND DIRECTIONS AND VERTICAL ION DRIFTS.

The direction of ion drift and the angle $\theta$ are deduced from equations (14-16). 
merge into one another, since the range of heights embraced by the figure (namely $1 \frac{1}{2}$ vertical wavelengths of the wind pattern) occupies a substantial height range in the real atmosphere, perhaps as much as $80 \mathrm{~km}$. The angle between the wind and current depends on $\mu$, as shown, but in all cases the current at a convergent null $(C)$ is equatorward, i.e. $j_{x}=0, j_{y}<0$. The same applies to the southern hemisphere on the assumption that the wind vector rotates in the opposite sense, as shown by the right hand column in Figure 1; in this case $j_{x}=0, j_{y}>0$ at any convergent null.

The assumption of no electric field is not justified, and can be removed by rewriting equations (15) and (16) in terms of "effective" wind components

$$
U_{x}^{\prime}=U_{x}+\left(E_{y} / B \sin I\right), \quad U_{y}^{\prime}=U_{y}-\left(E_{x} / B \sin I\right)
$$

and proceeding as before. Since the condition for $V_{i z}=0$ is now in terms of $U_{x}^{\prime}$ and $U_{y}^{\prime}$, the inclusion of the electric field alters the levels of the layer, if centered at the level $h_{\mathrm{i}} \bumpeq 130 \mathrm{~km}$, at which $\mu=1$ and where the Pedersen and Hall conductivities $\left(\sigma_{1}, \sigma_{2}\right)$ are both equal to $\frac{1}{2} N e / B$, has integrated layer conductivity given by

$$
\begin{gathered}
\Sigma_{1}, \Sigma_{2} \equiv \sigma_{1} \Delta h, \sigma_{2} \Delta h \\
=\left(\frac{1}{2} N e / B\right) \Delta h \cong 10^{-4} \Omega^{-1} \mathrm{~m}^{-1} \cdot 10^{3} \mathrm{~m}=0.1 \Omega^{-1}
\end{gathered}
$$

where we take $B=40 \mu \mathrm{T}$ for Arecibo. As the layer departs from the height $h_{i}$, either upward or downward, its Pedersen conductivity $\Sigma_{1}$ falls off, at first slowly but ultimately exponentially with scale height $\pm H$, according to the ordinary theory of ionospheric conductivity. The integrated Hali conductivity $\Sigma_{2}$ increases below $h_{\mathrm{i}}$ to a peak value twice that of equation (18), i.e. $0.2 \Omega^{-1}$, and falls off rapidly with height above $\dot{h}_{\mathrm{i}}$. These values may be compared with the $E$ - and $F$-region conductivities computed by Harper and Walker (1977) from Arecibo data for 16 nights at various seasons in 1974-1976, the minimum, average and maximum values being respectively as follows (Table 1):

Table 1. Night-time conductivities at Arecibo

\begin{tabular}{llcc}
\hline \multicolumn{1}{c}{ Conductivity $\left(\Omega^{-1}\right)$} & Minimum & Average & Maximum \\
\hline F-region Pedersen $(h>185 \mathrm{~km})$ & 0.016 & $0.1-0.2$ & 0.88 \\
E-region Pedersen $(95-185 \mathrm{~km})$ & 0.03 & $0.08-0.15$ & 0.25 \\
E-region Hall & 0.14 & $0.3-0.5$ & 0.91 \\
\hline
\end{tabular}

convergent nulls, but the electric current at those nulls is still equatorward. There are several complications to be considered in future work. The electric field, being height-independent, makes no contribution to the vertical convergence $\mathrm{d} V_{i} / \mathrm{d} h$ on which the detailed structure of the layer depends. It may, however, increase or diminish the current flowing at the nulls. If the electric field is too large in relation to the wind velocity, nulls may not exist at all. Without actual detailed experimental data, it does not seem worth enlarging on the possibilities here.

\section{ESTIMATE OF CONDUCTIVITY}

The directional properties of the layer currents can only be of significance if the conductivity within the layers is an appreciable fraction of the total night-time conductivity. Without attempting detailed calculations, we can assume that a typical night-time layer has a thickness $\Delta h=1 \mathrm{~km}$ and mean ion density $N=5 \times 10^{10} \mathrm{~m}^{-3}$. This typical
For average conditions, the contribution made by a single thin layer, namely $0.1 \Omega^{-1}$ as in equation (18), is marginal. However, there are sometimes several layers, sometimes thicker than $1 \mathrm{~km}$, and a value of $0.5 \Omega^{-1}$ is quite possible. With so much variability from night to night, we can only conclude that the directional effect of the layer currents may be significant on some individual nights, particularly if a well-developed layer exists around $130 \mathrm{~km}$ where its Pedersen conductivity will be greatest. We may remark that, should significant layers form in daytime by the same process (even with larger ion densities) their contribution to overall $E$-region conductivities of order $10 \Omega^{-1}$ is not likely to be significant.

From equations (10) and (11) we may deduce the conductivities that relate each current component to a component of electric field or neutral-air wind. To put each quantity in terms of eastward $(x)$ and northward $(y)$ components, we write $E_{y}=E_{n} \sin I$ (thereby taking $E_{\|}=0$ ) where $s=\sin I$ for brevity. 
The Pedersen-like conductivities are:

$$
\left.\begin{array}{r}
j_{x} / E_{x} \\
j_{y} s^{2} / E_{y} \\
-j_{x} / B U_{y} s \\
j_{y} s / B U_{x}
\end{array}\right\}=\frac{\mu N e / B}{1+\mu^{2}}
$$

The Hall-like conductivitıes are:

$$
\left.\begin{array}{c}
j_{x} s / E_{y} \\
-j_{y} s / E_{x} \\
j_{x} / B U_{x} \\
j_{y} / B U_{y}
\end{array}\right\}=\frac{N e / B}{1+\mu^{2}}
$$

\section{EFFECT ON $\boldsymbol{F}$-REGION MOTIONS}

The nocturnal $E$-region layers may influence $F$-region motion if their conductivity is sufficient to discharge $F$-region polarization fields to any extent. It is the Pedersen current that is important in this respect, the Hall currents being largely non-divergent and therefore relatively ineffective in discharging polarization fields. Consider first $F$-region drifts due to the polarization fields. The distribution of polarization charges and field is determined by the global pattern of tidal winds and magnetospheric sources, in accordance with the need to satisfy current continuity (div $\mathbf{j}=0$ ) everywhere in the ionosphere to a very high degree of approximation. The resulting field at Arecibo, say, is not likely to depend on local factors to any great extent. The existence of $E$-region layers with directional properties may have some influence on the global field distribution, but hardly an important one if the layers are localized and small, say a hundred kilometres or so in size. Since the tidal and magnetospheric sources are capable of driving substantial currents through the daytime ionosphere, with conductivities two orders of magnitude greater than those of the nocturnal layers [as estimated in equation (18)] it seems unlikely that the extra loading represented by nocturnal layers will have much effect. The most that could be expected is that meridionally-conducting layers, if sufficiently widespread, might slightly reduce the meridional polarization field as compared to the zonal field.

With thermospheric winds the situation is rather different. The $F$-region dynamo driven by such winds (Rishbeth, 1971a) is fairly easily short-circuited by the $E$-laycr. The effect of this shortcircuiting is to decouple zonal winds and ion drifts. In the equatorial zone the $F$-region dynamo acts strongly because of the geometry, and, with opencircuit conditions in the sub-equatorial $E$-region, zonal $F$-region winds and ion drifts are strongly coupled (Rishbeth, 1971b). This effect decreases away from the equator, with decreasing curvature of the field lines, but is still strong at Arecibo (dip $50^{\circ}$ ) according to the observations of Behnke and Hagfors (1974). Our analysis suggests that a nocturnal thin layer would help to decouple eastward winds and drifts, but have no effect on motions in other directions. The effect would depend on the horizontal extent of the thin layer. Studies of individual cases, some with and some without well-formed layers at around $130 \mathrm{~km}$, are clearly needed. The meridional wind structure at night over Arecibo is complex and interesting (Herrero and Meriwether, 1980; Burnside et al., 1981), and it appears that the $F$-region winds are dominated by meridional pressure gradients. The ion drifts, however, are probably dominated by zonal tidal fields and there is no obvious reason why these should be closely coupled to the winds.

\section{CONCLUSION}

We have drawn attention to the directional current-carrying properties of $E$-region layers formed at levels of zero vertical ion drift; we have shown that the current carried by such layers is purely equatorward and is essentially Pedersen current or Hall current, according as the layer is above or below about $130 \mathrm{~km}$; and we have suggested that individual case studies of $F$-region winds and drifts are needed to establish whether these $E$ region layers influence $F$-region dynamics by their electrical properties.

Acknowledgement-This research was stimulated by the observational work of R. M. Harper. J. C. G. Walker's work is supported in part by grant ATM 80-15316 from the Atmospheric Science Division, National Science Foundation. The National Astronomy and Ionosphere Center (Arecibo Observatory) is operated by Cornell University under contract with the National Science Foundation.

\section{REFERENCES}

Axford, W. I. (1961). Can. J. Phys. 39, 1393.

Axford, W. I. and Cunnold, D. M. (1966). Radio Sci. 1, 191.

Behnke, R. and Hagfors, T. (1974). Radio Sci. 9, 211.

Burnside, R. G., Herrero, F. A., Meriwether, J. R., Jr. and Walker, J. C. G. (1981). J. geophys. Res. 86, 5532.

Fujitaka, K., Ogawa, T. and Tohmatsu, T. (1971). I. atmos. terr. Phys, 33, 687.

Harper, R. M. (1977). J. geophys. Res. 82, 3245.

Harper, R. M. and Walker, J. C. G. (1977). Planet. Space Sci. 25, 197.

Harper, R. M., Wand, R. H. and Whitehead, J. D. (1975). Radio Sci. 10, 357.

Herrero, F. A. and Meriwether, J. R., Jr. (1980). $J$. geophys. Res. 85, 4191.

Macleod, M. A., Keneshea, T. J. and Narcisi, R. S. (1975). Radio Sci. 10, 371. 
Mathews, J. D. and Bekeny, F. S. (1979). J. geophys. Res. 84, 2743.

Miller, K. L. and Smith, L. G. (1978). J. geophys. Res. 83, 3761.

Rees, D., Dorling, E. B., Lloyd, K. H. and Low, C. (1976). Planet. Space Sci. 24, 475.

Rishbeth, H. (1971a). Planet. Space Sci. 19, 263.

Rishbeth, H. (1971b). Planet. Space Sci. 19, 357.
Rishbeth, H. and Garriott, O. K. (1969). Introduction to Ionospheric Physics. Academic Press, New York.

Rowe, J. F. (1974). J. atmos. terr. Phys. 36, 225.

Shen, J. S., Swartz, W. E., Farley, D. T. and Harper, R. M. (1976) J. geophys. Res. 81, 5517.

Trost, T.F. (1979). J. geophys. Res. 84, 2736.

Whitehead, J. D. (1961). J. atmos. terg. Phys. $20,49$.

Whitehead, J. D. (1970). Rev. geophys. Space Phys. 8, 65. 